\title{
I. I. Ogieveckij
}

Некоторые тауберовы теоремы винеровского типа для функции двух переменных

Czechoslovak Mathematical Journal, Vol. 8 (1958), No. 1, 76-85

Persistent URL: http://dml.cz/dmlcz/100278

\section{Terms of use:}

(C) Institute of Mathematics AS CR, 1958

Institute of Mathematics of the Czech Academy of Sciences provides access to digitized documents strictly for personal use. Each copy of any part of this document must contain these Terms of use.

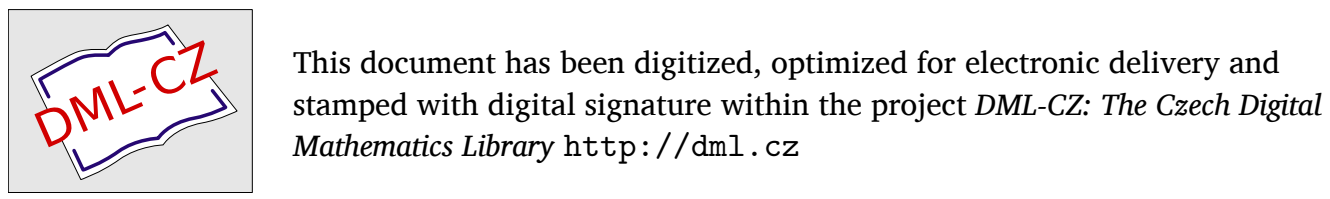




\title{
НЕКОТОРЫЕ ТАУБЕРОВЫ ТЕОРЕМЫ ВИНЕРОВСКОГО ТИПА ДЈЯ ФУНКЦИИ ДВУХ ПЕРЕМЕННЫХ*)
}

\author{
И. И. ОГИЕВЕЦКИЙ, Днепропетровск
}

(Поступило в редакцию 14/I 1957 г.)

В настоящей работе устанавливаются аналоги общих тауберовых теорем Н. В и нера для случая функций двух переменных и гаются некоторые их приложения к суммированию двойных рядов и интегралов. Применение указанных теорем дает возможность унифицированного рассмотрения ряда вопросов теории суммируемости двойных рядов и интегралов. Их значение в двумерной теории суммируемости основывается на том, что ряд известных в этой теории методов суммирования обладает ,,расщепляющимися“" ядрами, т. е. ядрами вида $K(x, y)=K_{1}(x) K_{2}(x)$.

Начнем с нескольких определений: Г'оворят, что функция $f(x)$ nрuнадлежит классу Винера [2] $(f \in W)$, если она $L$-интегрируема на всей бесконечной оси и её преобразование Фурье

$$
F(t)=\frac{1}{\sqrt{2 \pi}} \int_{-\infty}^{\infty} e^{i t u} f(u) \mathrm{d} u
$$

не обращается в нуль ни при каком действительном $t,-\infty<t<\infty$.

Функция $f(x, y)$ медленно колеблется в области - $-\infty<x<\infty,-\infty<$ $<y<\infty$, если

$$
\lim _{(x, y) \rightarrow \infty}\left|f\left(x^{\prime}, y^{\prime}\right)-f(x, y)\right|=0
$$

когда

$$
x^{\prime}>x, \quad y^{\prime}>y, \quad\left(x^{\prime}-x\right) \rightarrow 0, \quad\left(y^{\prime}-y\right) \rightarrow 0 .
$$

Функция $f(x, y)$ медленно колеблется в области $0<x<\infty, 0<y<\infty$, если

$$
\lim _{(x, y) \rightarrow \infty}\left|f\left(x^{\prime}, y^{\prime}\right)-f(x, y)\right|=0
$$

*) Результаты настоящей работы были частично сообщены на $I I I$ всесоюзном математическом съезде (25 июня-4 июля 1956 г., г. Москва), см. [1]. 
когда

$$
x^{\prime}>x, \quad y^{\prime}>y, \quad \frac{x^{\prime}}{x} \rightarrow 1, \quad \frac{y^{\prime}}{y} \rightarrow 1 .
$$

Теорема 1. ПIycmb

$$
\begin{gathered}
K(x, y)=K_{1}(x) K_{2}(y), \quad K_{1}(x) \epsilon W, \quad K_{2}(y) \varepsilon W, \\
K^{*}(x, y)=K_{1}^{*}(x) K_{2}^{*}(y), \quad K_{1}^{*}(x) \epsilon L(-\infty, \infty), \quad K_{2}^{*}(y) \epsilon L(-\infty, \infty), \\
|h(x, y)| \leqq M .
\end{gathered}
$$

Тогда из

$$
\lim _{(x, y) \rightarrow \infty} \int_{-\infty}^{\infty} K(x-u, y-v) h(u, v) \mathrm{d} u \mathrm{~d} v=s \int_{-\infty}^{\infty} K(u, v) \mathrm{d} u \mathrm{~d} v
$$

cледует

$$
\lim _{(x, y) \rightarrow \infty} \int_{-\infty}^{\infty} K^{*}(x-u, y-v) h(u, v) \mathrm{d} u \mathrm{~d} v=s \int_{-\infty}^{\infty} K^{*}(u, v) \mathrm{d} u \mathrm{~d} v .
$$

Доказательство. Из известной теоремы аппроксимации Винера [3] вытекает, что для любого $\varepsilon>0$ найдутся такие числа $m=m(\varepsilon), n=n(\varepsilon)$, $\xi_{\mu}(\mu=1,2, \ldots, m), \eta_{\nu}(\nu=1,2, \ldots, n)$, что

$$
\begin{aligned}
& K_{1}^{*}(x)=\sum_{\mu=1}^{m} a_{\mu} K_{1}\left(x+\xi_{\mu}\right)+\varphi_{1}(x), \\
& K_{2}^{*}\left((y)=\sum_{\nu=1}^{n} b_{\nu} K_{2}\left(y+\eta_{\nu}\right)+\varphi_{2}(y),\right.
\end{aligned}
$$

где

$$
\int_{-\infty}^{\infty}\left|\varphi_{1}(x)\right| \mathrm{d} x<\varepsilon, \int_{-\infty}^{\infty}\left|\varphi_{2}(x)\right| \mathrm{d} x<\varepsilon
$$

Отсюда

$$
\begin{gathered}
K_{1}^{*}(x-u) K_{2}^{*}(y-v)=\sum_{\mu=1, v=1}^{m, n} a_{\mu} b_{\nu} K_{1}\left(x-u+\xi_{\mu}\right) K_{2}\left(y-v+\eta_{v}\right)+ \\
+\varphi_{2}(y-v) \sum_{\mu=1}^{m} a_{\mu} K_{1}\left(x-u+\xi_{\mu}\right)+\varphi_{1}(x-u) \sum_{\nu=1}^{n} b_{\nu} K_{2}\left(y-v+\eta_{v}\right)+ \\
+\varphi_{1}(x-u) \varphi_{2}(y-v)
\end{gathered}
$$

и, значит,

$$
\int_{-\infty}^{\infty} K_{1}^{*}(x-u) K_{2}^{*}(y-v) h(u, v) \mathrm{d} u \mathrm{~d} v=\sum_{\mu=1, v=1}^{m, n} a_{\mu} b_{v} \int_{-\infty}^{\infty} K_{1}\left(x-u+\xi_{\mu}\right) .
$$

$. K_{2}\left(y-v+\eta_{v}\right) h(u, v) \mathrm{d} u \mathrm{~d} v+\int_{-\infty}^{\infty}\left\{\sum_{\mu=1}^{m} a_{\mu} K_{1}\left(x-u+\xi_{\mu}\right)\right\} \dot{\varphi}_{2}(y-v) h(u, v) \mathrm{d} u \mathrm{~d} v+$

$$
+\int_{-\infty}^{\infty}\left\{\sum_{v=1}^{n} b_{\nu} K_{2}\left(y-v+\eta_{v}\right)\right\} \varphi_{1}(x-u) h(u, v) \mathrm{d} u \mathrm{~d} v+
$$

$$
+\int_{-\infty}^{\infty} \varphi_{1}(x-u) \varphi_{2}(y-v) h(u, v) \mathrm{d} u \mathrm{~d} v .
$$


Для второго слагаемого из (2) имеем

$$
\left|\int_{-\infty}^{\infty}\left\{\sum_{\mu=1}^{m} a_{\mu} K_{1}\left(x-u+\xi_{\mu}\right)\right\} \varphi_{2}(y-v) h(u, v) \mathrm{d} u \mathrm{~d} v\right|<M(c+\varepsilon) \varepsilon,
$$

где

$$
\int_{-\infty}^{\infty}\left|K_{1}^{*}(x)\right| \mathrm{d} x \leqq c,
$$

и аналогичную оценку для третьего слагаемого. Легко видеть, что для четвертого слагаемого будет

$$
\left|\int_{-\infty}^{\infty} \varphi_{1}(x-u) \varphi_{2}(y-v) h(u, v) \mathrm{d} u \mathrm{~d} v\right|<M \varepsilon^{2} .
$$

Подставляя в (2) оценки (3) и (4), получим, что

$$
\begin{gathered}
\mid \int_{-\infty}^{\infty} K_{1}^{*}(x-u) K_{2}^{*}(y-v) h(u, v) \mathrm{d} u \mathrm{~d} v- \\
-\sum_{\mu=1, v=1}^{m, n} a_{\mu} b_{\nu} \int_{-\infty}^{\infty} K_{1}\left(x-u+\xi_{\mu}\right) K_{2}\left(y-v+\eta_{v}\right) h(u, v) \mathrm{d} u \mathrm{~d} v \mid< \\
<2 M(c+\varepsilon) \varepsilon+M \varepsilon^{2}<\delta
\end{gathered}
$$

при достаточно малом $\varepsilon$.

Из условия для функции $K(u, v)$ вытекает

$$
\begin{gathered}
\lim _{(x, y) \rightarrow \infty} \sum_{\mu=1, v=1}^{m, n} a_{\mu} b_{\nu} \int_{-\infty}^{\infty} K_{1}\left(x-u+\xi_{\mu}\right) K_{2}\left(y-v+\eta_{v}\right) h(u, v) \mathrm{d} u \mathrm{~d} v= \\
=s \sum_{\mu=1, v=1}^{m, n} a_{\mu} b_{v} \int_{-\infty}^{\infty} K_{1}(u) K_{2}(v) \mathrm{d} u \mathrm{~d} v= \\
=s \sum_{\mu=1, v=1}^{m, n} a_{\mu} b_{\nu} \int_{-\infty}^{\infty} K_{1}\left(u+\xi_{\mu}\right) K_{2}\left(v+\eta_{v}\right) \mathrm{d} u \mathrm{~d} v .
\end{gathered}
$$

Полагая в (1) $x=2 u, y=2 v$, получим

$$
\begin{gathered}
K_{1}^{*}(u) K_{2}^{*}(v)=\sum_{\mu=1, v=1}^{m, n} a_{\mu} b_{v} K_{1}\left(u+\xi_{\mu}\right) K_{2}\left(v+\eta_{v}\right)+ \\
+\varphi_{2}(v) \sum_{\mu=1}^{m} a_{\mu} K_{1}\left(u+\xi_{\mu}\right)+\varphi_{1}(u) \sum_{v=1}^{n} b_{v} K_{2}\left(v+\eta_{v}\right)+ \\
+\varphi_{1}(u) \varphi_{2}(v) .
\end{gathered}
$$

Умножая (5b) на $s$, интегрируя пго переменным $u$ и $v$ в пределах $-\infty<u<\infty,-\infty<v<\infty$ и используя оценки, аналогичные и (4), получим

$$
\begin{aligned}
& \mid s \sum_{\mu=1, v=1}^{m, n} a_{\mu} b_{\nu} \int_{-\infty}^{\infty} K_{1}\left(u+\xi_{\mu}\right) K_{2}\left(v+\eta_{v}\right) \mathrm{d} u \mathrm{~d} v- \\
& -s \int_{-\infty}^{\infty} K_{1}^{*}(u) K_{2}^{*}(v) \mathrm{d} u \mathrm{~d} v \mid<2 s(c+\varepsilon) \varepsilon+s \varepsilon^{2}<\delta
\end{aligned}
$$

при достаточно малом $\varepsilon$. 
Сопоставляя (5), (5a) и (6), приходим к утверждению теоремы, т. к. $\delta$ может быть взято произвольно малым.

Положим (см. [2])

$$
\begin{gathered}
e^{x}=x^{\prime}, \quad e^{y}=y^{\prime}, \quad e^{u}=u^{\prime}, \quad e^{v}=v^{\prime}, \\
L_{1}\left(e^{x}\right)=e^{-x} K_{1}(-x), \quad L_{2}\left(e^{y}\right)=e^{-y} K_{2}(-y)
\end{gathered}
$$

и от переменных $x, y, u$ и $v$ перейдем к переменным $x^{\prime}, y^{\prime}, u^{\prime}, v^{\prime}$. При этой замене функции, заданные при - $<x<\infty,-\infty<y<\infty$, перейдут в функции, заданные в $0<x^{\prime}<\infty, 0<y^{\prime}<\infty$, п класс Винера $W$ функций $f(x)$ превратится в класс $W_{0}$ функций $F(x) \in L(0, \infty)$, для которых $\int_{0}^{\infty} F(u) u^{-i x} \mathrm{~d} u \neq 0$ при любом действительном $x$.

Теореме I будет соответствовать следующая

Теорема 2. Пусть

$$
\begin{gathered}
L(x, y)=L_{1}(x) L_{2}(y), \quad L_{1}(x) \epsilon W_{0}, \quad L_{2}(y) \epsilon W_{0} ; \\
L^{*}(x, y)=L_{1}^{*}(x) L_{2}^{*}(y), \quad L_{1}^{*}(x) \epsilon L(0, \infty), \quad L_{2}^{*}(y) \epsilon L(0, \infty) .
\end{gathered}
$$

и $h(x, y)$ ограничено; тогда из

$$
\lim _{[x, y) \rightarrow \infty} \frac{1}{x y} \int_{0}^{\infty} L\left(\frac{u}{x}, \frac{v}{y}\right) h(u, v) \mathrm{d} u \mathrm{~d} v=s \int_{0}^{\infty} L(u, v) \mathrm{d} u \mathrm{~d} v
$$

следует

$$
\lim _{(x, y) \rightarrow \infty} \frac{1}{x y} \int_{0}^{\infty} L^{*}\left(\frac{u}{x}, \frac{v}{y}\right) h(u, v) \mathrm{d} u \mathrm{~d} v=s \int_{0}^{\infty} L^{*}(u, v) \mathrm{d} u \mathrm{~d} v .
$$

Определим теперь $L^{*}(x)=L_{1}^{*}(x)=L_{2}^{*}(x)$ следующим образом

$$
L_{1}^{*}(x)=\left\langle\begin{array}{ll}
1, & x<1 \\
0, & x>1
\end{array} .\right.
$$

Легко видеть, что $L^{*}(x)$ удовлетворяет условиям теоремьі 2.

Пусть $h(x, y)$ медленно колеблется (см. выше) в области $0<x<\infty$, $0<y<\infty$. Тогда, как нетрудно установить, следуя рассуждениям $\mathrm{K}$. Кноппа из [4],

$$
\lim _{(x, y) \rightarrow \infty} h(x, y)=s,
$$

если

$$
\lim _{(x, y) \rightarrow \infty} \frac{1}{x y} \int_{0}^{x} \int_{0}^{y} h(u, v) \mathrm{d} u \mathrm{~d} v=s .
$$

Используя теорему 2 с $L^{*}(x)=L_{2}^{*}(x)=L_{1}^{*}(x)$ в (6а) и только что указанный факт, приходим к следующей 


\section{Теореме 3. Ecли}

$$
L(x, y)=L_{1}(x) L_{2}(y), \quad L_{1}(x) \epsilon W_{0}, \quad L_{2}(y) \epsilon W_{0}, \quad h(x, y)
$$

ограничено и медленно колеблется в области $0<x<\infty, 0<y<\infty$, то из

$$
\lim _{(x, y) \rightarrow \infty} \frac{11}{x y} \int_{0}^{\infty} L\left(\frac{u}{x}, \frac{v}{y}\right) h(u, v) \mathrm{d} u \mathrm{~d} v=s \int_{0}^{\infty} L(u, v) \mathrm{d} u \mathrm{~d} v
$$

вытекает, что

$$
\lim _{(x, y) \rightarrow \infty} h(x, y)=s .
$$

Совершая в последней теореме переход к переменным; обратный тому, который производился при получении теоремы 2 из теоремы 1 , приходим к следующему результату:

\section{Теорема 4. Ecли}

$$
K(x, y)=K_{1}(x) K_{2}(y), \quad K_{1}(x) \epsilon W, \quad K_{2}(y) \epsilon W,
$$

$h(x, y)$ ограничено и медленно колеблется в интервале $-\infty<x<\infty$, $-\infty<y<\infty$ и

$$
\lim _{(x, y) \rightarrow \infty} \int_{-\infty}^{\infty} K(x-u, y-v) h(u, v) \mathrm{d} u \mathrm{~d} v=s \int_{-\infty}^{\infty} K(u, v) \mathrm{d} u \mathrm{~d} v
$$

To

$$
\lim _{(x, y) \rightarrow \infty} h(x, y)=s .
$$

Перейдем к приложениям установленных выше теорем. Докажем, например, теорему Кноппа [4].

Теорема Кноппа. Если последовательность $s_{m n}$ ограничена и суммируется методом Абеля $k s$, то она такмсе суммируется методом средне арифметических $к s$.

Действительно, определив функцию $h(x, y)$ следующцм образом

$$
h(x, y)=s_{m n} \quad \text { для } \quad m \leqq x<m+1, \quad n \leqq y<n+1
$$

и полагая $u=e^{-\frac{1}{x}}, v=e^{-\frac{11}{y}}$, мы можем написать

$$
\begin{gathered}
\lim _{(u, v) \rightarrow \mathbf{1}}(1-u)(1-v) \sum_{m=0, n=0}^{\infty} s_{m n} u^{m} v^{n}= \\
=\lim _{(\boldsymbol{x}, \boldsymbol{y}) \rightarrow \infty}\left(1-e^{-\frac{\mathbf{i}^{1}}{x}}\right)\left(1-e^{-\frac{1}{y}}\right) \sum_{m=0, n=0}^{i \infty} s_{m n} e^{-\frac{m}{x}} e^{-\frac{n}{y}}= \\
=\lim _{(x, y) \rightarrow \infty} \sum_{m=0, n=0}^{\infty} s_{m n}\left(e^{-\frac{m}{x}}-e^{-\frac{(m+1)}{x}}\right)\left(e^{-\frac{n}{i^{y}}}-e^{-\frac{(n+1)}{y}}\right)=
\end{gathered}
$$




$$
\begin{gathered}
=\lim _{(x, y) \rightarrow \infty} \frac{1}{x y} \sum_{m=0, n=0}^{\infty} s_{m n} \int_{m}^{m+1} e^{-\frac{u}{x}} \mathrm{~d} u \int_{n}^{n+1} e^{--\frac{v}{y}} \mathrm{~d} v= \\
=\lim _{(x, y) \rightarrow \infty} \frac{1}{x y} \int_{0}^{\infty} e^{--\frac{u}{x}} e^{-\frac{v}{y}} h(u, v) \mathrm{d} u \mathrm{~d} v=s,
\end{gathered}
$$

т. к. последовательность преднолагается суммируемой методом Абеля.

Используя вторую теорему с $L_{1}(z)=L_{2}(z)=e^{-z}$ и с $L_{1}^{*}(z)=L_{2}^{*}(z)=\mathrm{l}$ для $z<1$ и с $L_{1}^{*}(z)=L_{2}^{*}(z)=0$ для $z>1$, получим, что функция $h(x, y)$, а, следовательно, и последовательность $s_{m n}$, суммируется методом среднеарифметических к $s$, что и тр. цоказать.

Покажем, как используя теорему 4, можно прийти к следующему результату, также содержашемуся в [4].

Теорема. Если последовательность $s_{m n}$ ограничена, суммируетсл метоэом Абеля к s и медленно колеблется (Опр. медлениого колебания двойноӥ последовательности см. в [4]), то

$$
\lim _{(m, n) \rightarrow \infty} s_{m n}=s .
$$

Действительно, лекко видеть, что из условий теоремы вытекает спра- . ведливость (7); т. к. $s_{m n}$ медленно колеблется, то отсюда вытекает, что $h(x, y)$ также медленно колеблется, и следовательно, непосредственно используя теорему 3 с $L_{1}(z)=L_{2}(z)=e^{-z}$, получим, что

$$
\lim _{(m, n) \rightarrow \infty} s_{m n}=s \text {. }
$$

Доказательство этого результата, построенное на совершенно других соображениях, содержится также в работе Деланжа [5].

Рассмотрим некоторые применения к суммированию интеграғтов методом Чезаро.

По аналогии с одномерным случаем говорят, что функция $s(x, y)$ суммируется методом $(C, \alpha, \beta) \alpha>0, \beta>0$ к значению $s$, если

$$
\lim _{(x, y) \rightarrow \infty} \bar{\sigma}^{\alpha} \beta(x, y, s(x, y))=s
$$

где

$$
\bar{\sigma}^{\alpha, \beta}(x, y, s(x, y))=\frac{\alpha \beta}{x^{\alpha} y^{\beta}} \int_{0}^{x} \int_{0}^{y}(x-u)^{\alpha-1}(y-v)^{\beta-1} s(u, v) \mathrm{d} u \mathrm{~d} v
$$

и, что интеграл $\int_{0}^{\infty} f(u, v) \mathrm{d} u \mathrm{~d} v$ суммируется методом $(C, \alpha, \beta)$ к $s$, если

$$
\lim _{(x, y) \rightarrow \infty} \sigma^{\alpha} \beta(x, y, f)=s,
$$


где

$$
\sigma^{\alpha, \beta}(x, y, f)=\int_{0}^{x} \int_{0}^{y}\left(1-\frac{u}{x}\right)^{\alpha}\left(1-\frac{v}{y}\right)^{\beta} f(u, v) \mathrm{d} u \mathrm{~d} v .
$$

Последнее выражение имеет смысл при $\alpha>-1, \beta>-1$. Из (8) вытекает, что

$$
\begin{gathered}
\bar{\sigma}^{\alpha+\eta, \beta+\delta}(x, y, s(x, y))=\frac{\Gamma(\alpha+\eta+1) \Gamma(\beta+\delta+1)}{\Gamma(\eta) \Gamma(\delta) \Gamma(\alpha+1) \Gamma(\beta+1)} \frac{1}{x y} \int_{0}^{x} \int_{0}^{y}\left(1-\frac{u}{x}\right)^{\eta-1} . \\
.\left(1-\frac{v}{y}\right)^{\delta-1}\left(\frac{u}{x}\right)^{\alpha}\left(\frac{v}{y}\right)^{\beta} \bar{\sigma}^{\alpha, \beta}(u, v, s) \mathrm{d} u \mathrm{~d} v .
\end{gathered}
$$

Точно такая же формула имеет место и для $\sigma^{\alpha, \beta}(x, y, f)$.

Нетрудно видеть, что ядро суммирования в (10), имеющее вид $L_{3}(u) L_{2}(v)$, где

$$
L_{1}(z)=\frac{\Gamma(\alpha+\eta+1)}{\Gamma(\eta) \Gamma(\alpha+1)}(1-z)^{\eta-1} z^{\alpha} \quad \text { для } \quad z<1 \text { и } L_{1}(z)=0 \quad \text { для } \quad z>1
$$

и

$$
\begin{gathered}
L_{2}(z)=\frac{\Gamma(\beta+\delta+1)}{\Gamma(\delta) \Gamma(\beta+1)}(1-z)^{\delta-1} z^{\beta} \text { для } z<1 \\
\text { и } L_{2}(z)=0 \text { для } z>1,
\end{gathered}
$$

принадлежит классу $W_{0}$.

Рассмотрим некоторые приложения установленных выше теорем к суммированию интегралов. Покажем, что из теоремы 2 непосредственно вытекает следующая теорема, характеризующая свойства выпуклости суммирования интегралов методом Чезаро.

Теорема. Если $\partial л я \int_{0}^{\infty} f(u, v) \mathrm{d} u \mathrm{~d} v$.

$$
\left|\sigma^{\alpha, \beta}(x, y, f)\right| \leqq c
$$

при всех $x>0, y>0$ и этот интеграл суммируетсл методом Уезаро порядка $(C, \alpha+\eta, \beta+\delta) \kappa s$, где $\eta>0, \delta>0-$ некоторые фиксированные числа, то он такме суммируется методом $\left(C, \alpha^{\prime}, \beta^{\prime}\right) \kappa s$ при любых $\alpha^{\prime}>\alpha>-1, \beta^{\prime}>\beta>-1$.

Действительно, из условий теоремы вытекает, что

$$
\lim _{(x, y) \rightarrow \infty} \frac{1}{x y} \int_{0}^{x} \int_{0}^{y} L_{1}\left(\frac{u}{x}\right) L_{2}\left(\frac{v}{y}\right) \sigma^{\alpha, \beta}(u, v, f) \mathrm{d} u \mathrm{~d} v=s
$$


при данных $\eta>0, \delta>0$, где $L_{1}(z)$ и $L_{2}(z)$, определены в (11). Так как $L_{1}(z)$ и $L_{2}(z)$ принадлежит классу $W_{0}$ при любых $\eta>0$ и $\delta>0$, то применяя теорему 2 к (10a), приходим к утверждению теоремы.

Несколько варьируя предыдущие рассуждения, легко приходим к теореме включения для суммирования интегралов методом Чезаро.

Теорема. Если $\partial л s \int_{0}^{\infty} f(u, v) \mathrm{d} u \mathrm{~d} v$

$$
\left|\sigma^{\alpha \cdot \beta}(x, y, f)\right| \leqq c
$$

при $\quad x \geqq 0, y \geqq 0$ и этот интеграл суммируется методом $(C, \alpha, \beta)$, $\alpha>-1, \beta>-1, k s$, то он также суммируетсл методом $\left(C, x^{\prime}, \beta^{\prime}\right)$, $\alpha^{\prime}>\alpha, \beta^{\prime}>\beta$ к тому же значению.

Действительно, из условий теоремы следует, что $\sigma^{\alpha} \beta(x, y, f)$ суммируется методом средне-арифметических. Но ядро метода средне-арифметических принадлежит классу $W_{0}$. Поэтому, применяя теорему 2 к $(10)$, получим, что $\lim \sigma^{\alpha+\eta} \beta+\delta(x, y, f)=s$ при любых $\eta>0, \delta>0$.

$(x, y) \rightarrow \infty$

Другое доказательство предыдущих двух теорем см. в [6].

Другой иллюстрацией применения теоремы 2 может служить доказательство следуюшей теоремы, являюпейся непрерывным аналогом первой теоремы из [7].

Теорема. Eсли $\int_{0}^{\infty} f(u, v) \mathrm{d} u \mathrm{~d} v$ суммируется методом $(C, \alpha+\eta, \beta+\delta)$ $\kappa s и$ его $\sigma^{\alpha, \beta}(x, y, f)$ средние ограничены, то әти средние суммируются методом $(C, \eta, \delta), \eta>0, \delta>0 \kappa s$.

Из условий теоремы, используя первую из предшествующих двух теорем, заключаем, что $\int_{0}^{\infty} f(u, v) \mathrm{d} u \mathrm{~d} v$ суммируется методом $(C, \alpha+1, \beta+1)$ к $s$. Полагая, затем $\eta=\delta=1$ в (10) получим

$$
\sigma^{\alpha+1, \beta+1}(x, y, f)=\frac{(\alpha+1)(\beta+1)}{x y} \int_{0}^{x} \int_{0}^{y}\left(\frac{u}{x}\right)^{\alpha}\left(\frac{v}{y}\right)^{\beta} \sigma^{\alpha, \beta}(u, v, f) \mathrm{d} u \mathrm{~d} v,
$$

где ядро перехода от $\boldsymbol{\sigma}^{\alpha, \beta}(x, y, f)$ к $\sigma^{\alpha+1, \beta+1}(x, y, f)$ имеет вид $L_{1}(x) L_{2}(x)$ с $L_{1}(z)=(\alpha+1) z^{\alpha}$ при $z<1$ и $L_{1}(z)=0$ при $z>1$, а $L_{2}(z)=(\beta+1) z^{\beta}$ при $z<1$ и $L_{2}(z)=0$ при $z>1$.

Нетрудно видеть что $L_{1}(z)$ и $L_{2}(z)$ принадлежат классу $W_{0}$ Винера.

Полагая в $(10) \alpha=\eta, \beta=\delta$ и $s(x, y)=\sigma^{\alpha, \beta}(x, y, f)$ получим

$$
\bar{\sigma}^{\eta, \delta}\left(x, y, \sigma^{\alpha, \beta}(x, y, f)\right)=\frac{\eta \delta}{x y} \int_{0}^{x} \int_{0}^{y}\left(1-\frac{u}{x}\right)^{\eta-1}\left(1-\frac{v}{y}\right)^{\delta-1} \sigma^{\alpha, \beta}(u, v, f) \mathrm{d} u \mathrm{~d} v
$$


т. к. $\lim \sigma^{\alpha+1, \beta+1}(x, y, f)=s$, то, применяя к (12) теорему 2, заключаем, $(x, y) \rightarrow \infty$

что предел правой части $(13)$ при $(x, y) \rightarrow \infty$ существует и равен $s$. Следовательно $(C, \alpha, \beta)$ - средние интеграла $\int_{0}^{\infty} f(u, v) \mathrm{d} u \mathrm{~d} v$ суммируются методом $(C, \eta, \delta) \eta>0, \delta>0$ к $s$.

\section{ЛИТЕРАТУРА}

[1] И.И. Огиевецкий: K теории суммирования кратных числовых рядов, Труды третьего всесоюзного математического съезда, Том. 1 (1956), стр. 94.

[2] Г. Харди: Расходящиеся ряды, Москва 1951.

「3] N. Wiener: Tauberian theorems, Annals of Mathematics 33 (1932), 1-100.

[4] K. Knopp: Limitierungs-Umkehrsätze für Doppelfolgen, Math. Z. 45 (1939), 573-589.

[5] H. Delange: Théorèmes taubèriens pour les" séries multiples de Dirichlet et les intégrales multiples de Laplace, Annales de l'école Normale 70 (1953), 51 - 103.

[6] М.Ф. Тиман: Диссертация - Днепропетровский Гос. Унив., г. Днепропетровск 1952.

[7] И. И. Огиевецкий: О суммировании кратных рядов, ДАН СССР 95 (1954), 713-716.

\section{Summary \\ SOME TAUBERIAN THEOREMS OF N. WIENER'S TYPE FOR FUNCTIONS OF TWO VARIABLES}

\section{I. OGIEVECKIJ, Dniepropetrovsk \\ (Received January 14, 1957)}

The following theorems are analogous to the well-known tauberian theorems of N. Wiener.

Theorem 1. Let $W$ be the class of Wiener functions. Let $K(x, y)=K_{1}(x) K_{2}(y)$ where $K_{1}, K_{2} \in W$. Let $K^{*}(x, y)=K_{1}^{*}(x) K_{2}^{*}(y)$ where $K_{1}^{*}, K_{2}^{*} \in L(-\infty,+\infty)$. Let $h(x, y)$ by a bounded function.

Then the relation

$$
\lim _{(x, y) \rightarrow \infty} \int_{-\infty}^{\infty} K(x-u, y-v) h(u, v) \mathrm{d} u \mathrm{~d} v=s \int_{-\infty}^{\infty} K(u, v) \mathrm{d} u \mathrm{~d} v
$$

implies

$$
\lim _{(x, y) \rightarrow \infty} \int_{-\infty}^{\infty} K^{*}(x-u, y-v) h(u, v) \mathrm{d} u \mathrm{~d} v=s \int_{-\infty}^{\infty} K^{*}(u, v) \mathrm{d} u \mathrm{~d} v .
$$


Theorem 2. Let $W_{0}$ be the class of Wiener functions in $(0, \infty)$. Let $L(x, y)=$ $=L_{1}(x) L_{2}(y)$ where $L_{1}, L_{2} \epsilon W_{0}$. Let $L^{*}(x, y)=L_{1}^{*}(x) L_{2}^{*}(y)$, where $L_{1}^{*}, L_{2}^{*} \epsilon$ $\epsilon L(0, \infty)$. Let $h(x, y)$ be a bounded function.

Then the relation

$$
\lim _{(x, y) \rightarrow \infty} \frac{1}{x y} \int_{0}^{\infty} L\left(\frac{u}{x}, \frac{v}{y}\right) h(u, v) \mathrm{d} u \mathrm{~d} v=s \int_{0}^{\infty} L(u, v) \mathrm{d} u \mathrm{~d} v
$$

implies

$$
\lim _{(x, y) \rightarrow \infty} \frac{1}{x y} \int_{0}^{\infty} L^{*}\left(\frac{u}{x}, \frac{v}{y}\right) h(u, v) \mathrm{d} u \mathrm{~d} v=s \int_{0}^{\infty} L^{*}(u, v) \mathrm{d} u \mathrm{~d} v .
$$

Theorem 3. Let $L(x, y)$ be defined as in 2. Let $h(x, y)$ be bounded and slowly oscillating in $0<x<\infty, 0<y<\infty$.

Then the relation

$$
\lim _{(x, y) \rightarrow \infty} \frac{1}{x y} \int_{0}^{\infty} L\left(\frac{u}{x}, \frac{v}{y}\right) h(u, v) \mathrm{d} u \mathrm{~d} v=s \int_{0}^{\infty} L(u, v) \mathrm{d} u \mathrm{~d} v
$$

implies

$$
\lim _{(x, y) \rightarrow \infty} h(x, y)=s
$$

Theorem 4. Let $K(x, y)$ be defined as in 1. Let $h(x, y)$ by bounded and slowly oscillating in $-\infty<x<\infty,-\infty<y<\infty$.

Then the relation

$$
\lim _{(x, y) \rightarrow \infty} \int_{-\infty}^{\infty} K(x-u, y-v) h(u, v) \mathrm{d} u \mathrm{~d} v=s \int_{-\infty}^{\infty} K(u, v) \mathrm{d} u \mathrm{~d} v
$$

implies

$$
\lim _{(x, y) \rightarrow \infty} h(x, y)=s .
$$

The proof is based on the "closure of translations theorem" of $\mathrm{N}$. Wiener and a generalization of a result of K. KNOPP. These theorems enable us to consider from one point of view many problems of summability of double series and integrals. The importance of these theorems for two-dimensional summability is based on the fact that many known summability methods have ,factorable kernels" $K(x, y)=K_{1}(x) K_{2}(y)$.

We also consider some applications of the theorems proved. 